\title{
BMJ Predictors of good functional outcome Open in counterpulsation-treated recent ischaemic stroke patients
}

\author{
Wenhua Lin, Jinghao Han, Xiangyan Chen, Li Xiong, Ho Wan Leung, \\ Thomas W Leung, Yannie Soo, Lawrence Ka Sing Wong
}

To cite: Lin W, Han J, Chen $\mathrm{X}$, et al. Predictors of good functional outcome in counterpulsation-treated recent ischaemic stroke patients. BMJ Open 2013;3: e002932. doi:10.1136/ bmjopen-2013-002932

- Prepublication history for this paper is available online. To view these files please visit the journal online (http://dx.doi.org/10.1136/ bmjopen-2013-002932).

Received 22 March 2013 Accepted 20 May 2013

This final article is available for use under the terms of the Creative Commons Attribution Non-Commercial 2.0 Licence; see http://bmjopen.bmj.com
Division of Neurology, Department of Medicine and Therapeutics, The Chinese University of Hong Kong, Hong Kong

\section{Correspondence to} Professor Lawrence Ka Sing Wong; ks-wong@cuhk.edu.hk

\section{ABSTRACT}

Objectives: External counterpulsation (ECP) is a non-invasive method being investigated for ischaemic stroke. We aimed to explore predictors of good functional outcome for ECP-treated ischaemic stroke patients who completed a minimum of 10 sessions.

Methods: We analysed our ECP registry of ischaemic stroke patients with cerebral large artery stenosis who underwent ECP therapy at the Prince of Wales Hospital from 2004 to 2010. We included 155 patients who completed at least 10 sessions of ECP and had 3month follow-up data as well as 52 medical controls. Functional outcomes were dichotomised into good outcome (modified Rankin Scale (mRS) 0-2) and bad outcome (mRS 3-6). We compared the differences in two groups in terms of demographics, medical history and parameters of ECP treatment.

Results: At 3 months after stroke, $70.5 \%$ of patients who finished the whole course of ECP had a good outcome (only $46.5 \%$ in the unfinished group and $38.5 \%$ in the medical group). Among all 207 recruited cases, $119(57.5 \%)$ patients had a good outcome at 3 months after stroke. Compared with the bad outcome group, patients in the good outcome group were younger and had a lower baseline National Institutes of Health Stroke Scale (NIHSS) and longer ECP therapy duration. Multivariate logistic regression showed that ECP duration (OR 1.032), baseline NIHSS (OR 0.734) and age (OR 0.961) were independent predictors for a favourable outcome.

Conclusions: Duration of ECP therapy is first found to be an important predictor for good outcome of ECPtreated ischaemic stroke patients, in addition to the well-known prognostic factors such as age and NIHSS.

\section{INTRODUCTION}

External counterpulsation (ECP) is a noninvasive and effective method for augmenting the cerebral blood flow of ischaemic stroke patients with large artery stenosis. ${ }^{1}$ Nowadays, the commonly used ECP device is a sequential pneumatic system with inflatable cuffs, called enhanced external counterpulsation (EECP), which was developed in 1980s. ${ }^{2}$

\section{ARTICLE SUMMARY}

Article focus

- We aim to explore the effects of external counterpulsation (ECP) on ischaemic stroke from an ECP registry.

- What are the significant predictors of good functional outcome for ECP-treated ischaemic stroke patients?

Key messages

- We included 155 patients who completed at least 10 sessions of ECP as well as 52 medical controls, and followed up their functional outcomes at 3 months after recruitment.

- We compared the differences in the good outcome and bad outcome groups in terms of demographics, medical history and parameters of ECP treatment.

- Duration of ECP therapy is first found to be an important predictor for good outcome of ECP-treated ischaemic stroke patients, in addition to the well-known prognostic factors such as age and National Institutes of Health Stroke Scale.

Strengths and limitations of this study

- This is the first report from an ECP registry for ischaemic stroke patients.

- For the first time, the duration of ECP therapy is identified as an independent predictor of stroke recovery after ECP treatment.

- This is a retrospective study, and ECP treatment is not randomised.

- Our sample size is relatively small.

In the EECP system, there are three pairs of pneumonic cuffs applied to the calves, lower thighs and upper thighs. The ECG triggers cuff inflation sequentially from distal to proximal during diastole and release cuff pressure before the start of systole. Diastolic pressure on the lower extremities improves venous return and cardiac output, while deflation before systole leads to increased systolic unloading. Therefore, ECP could help to increase perfusion of vital organs, such as the brain, liver and kidney. ${ }^{3}$ Today, EECP is 
widely accepted as a safe and highly beneficial treatment for angina pectoris. ${ }^{5}$ The standard duration of EECP treatment is generally several weeks ( 5 daily $1 \mathrm{~h}$ sessions each week for 7 weeks, for a total of 35 sessions), based on empiric data from studies in China. ${ }^{6}$ A short course of 10 sessions of EECP therapy before a high-risk coronary artery bypass graft could improve myocardial perfusion and left ventricular function. ${ }^{7}$ Currently, ECP is a new treatment concept for ischaemic stroke and the investigation of its safety for acute ischemic stroke patients is undergoing in a clinical trial (Safety Study of External Counterpulsation as a Treatment for Acute Ischemic Stroke, CUFFS, NCT00983749). Our previous study found that ECP may help the recovery of ischaemic stroke patients with large artery occlusive disease. ${ }^{8}$

Previous reports have used data from the EECP registry to investigate various effects of EECP. ${ }^{9}{ }^{10}$ We aim to explore the effects of ECP on ischaemic stroke from our ECP registry, which includes stroke patients only. The purpose of this study is to discover the predictors of good functional outcome for ECP-treated ischaemic stroke patients who completed treatment for a minimum of 10 session. We proposed to find out retrospectively the significant predictors in different outcome groups and then identify those independent factors in a multivariate model.

\section{MATERIALS AND METHODS}

\section{Subjects}

We recruited consecutive patients with recent ischaemic stroke associated with cerebral large artery stenosis who were hospitalised at the Prince of Wales Hospital, Hong Kong. Acute ischaemic stroke was diagnosed according to the WHO criteria, and patients were included in the study if there was at least one carotid or cerebral large artery stenosis (moderate stenosis or $>50 \%$ stenosis) diagnosed by MR angiography, CT angiography, transcranial Doppler ${ }^{11}$ or Doppler duplex. Those with cardioembolic stroke or a history of intracranial haemorrhage were excluded. Patients were excluded if their cerebral large artery stenosis was caused by vasculitis. As for patients with stroke due to small vessel occlusion, the patient was included if he had imaging evidences of concurrent cerebral large artery stenosis, otherwise excluded. Contraindications for ECP (sustained hypertension, aortic aneurysm, severe peripheral artery disease and carotid dissection), severe systemic diseases and malignancy were also in the exclusion criteria. The study was approved by the local medical ethics committee (Joint CUHK-NTEC Clinical Research Ethics Committee). Patients who agreed to receive ECP treatment as an additional therapy for their routine medical treatment were given ECP therapy. Among those stroke patients recruited from 3 May 2004 to 15 April 2010, 226 received ECP treatment using the Enhanced External Counterpulsation system, Vamed Medical Instrument Company device, model number MC2 or MC3 (Guangdong, China, cuff inflation pressure between 150 and $225 \mathrm{~mm} \mathrm{Hg}$ ). The standard protocol involved $351 \mathrm{~h}$ sessions (at least 5 times/week), but some failed to complete the whole course treatment for reasons as described below. Since most patients (188 cases, 83.2\%) completed at least 10 hourly sessions of ECP and 10 sessions of EECP therapy were suggested to be beneficial to surgical outcome after coronary artery bypass graft, ${ }^{7}$ we included these 188 patients in the next step as shown in figure 1. We followed up patients at 3 months after treatment with the modified Rankin Scale (mRS) at clinic visits; 155 patients successfully completed follow-up while 33 did not. Among those 155 patients, 112 completed all 35 sessions (finished ECP group) and 43 did not (unfinished ECP group). Of the 43 patients who did not receive all 35 sessions of ECP treatment, $16(37.2 \%)$ failed to complete the whole course because of lack of social support for outpatient attendance after discharge from the hospital. Eight patients $(18.6 \%)$ declined further treatment after their neurological deficit improved. Four patients $(9.3 \%)$ discontinued treatment because they could not tolerate the adverse effects, such as leg pain, haematuria and skin abrasion. Seven patients $(16.3 \%)$ stopped treatment because of other comorbidities such as depression, ventricular ectopic beat and renal failure. Six patients (14\%) declined to continue treatment for personal reasons and two patients for reasons unknown. At the 3-month follow-up, one patient developed cardiovascular disease, and the other died from renal failure (neither completed 35 sessions of ECP). No patients had transient ischaemic attack (TIA) or recurrent stroke.

We also recruited 52 hospitalised ischaemic stroke patients during May 2004 and April 2010, who fulfilled the same inclusion and exclusion criteria with the ECP group but refused ECP and received only medical treatment. Assessment of mRS at 3 months after stroke was performed at their clinical visits as well. All recruited patients signed informed consent.

\section{Data analysis}

We dichotomised the 3-month follow-up mRS into good outcome (mRS 0-2) and bad outcome (mRS 3-6). We compared the demographic differences (eg, age, gender and vascular risk factors) between two groups, as well as the medical history, medications and the number of ECP sessions completed. Continuous data were analysed by independent-sample $t$ tests when there was a normal distribution and by the Mann-Whitney test if there was a skewed distribution. Category data were analysed by the $\chi^{2}$ test. We used multivariate logistic regression to identify the independent predictors for a better outcome among these ECP-treated stroke patients. Significance level was defined as $\mathrm{p}<0.05$.

\section{RESULTS}

There were 207 ischaemic stroke patients recruited in the analysis, of which 155 received ECP and 52 did not. 
Figure 1 Flow chart of external counterpulsation (ECP)-treated patient selection from the ECP registry.

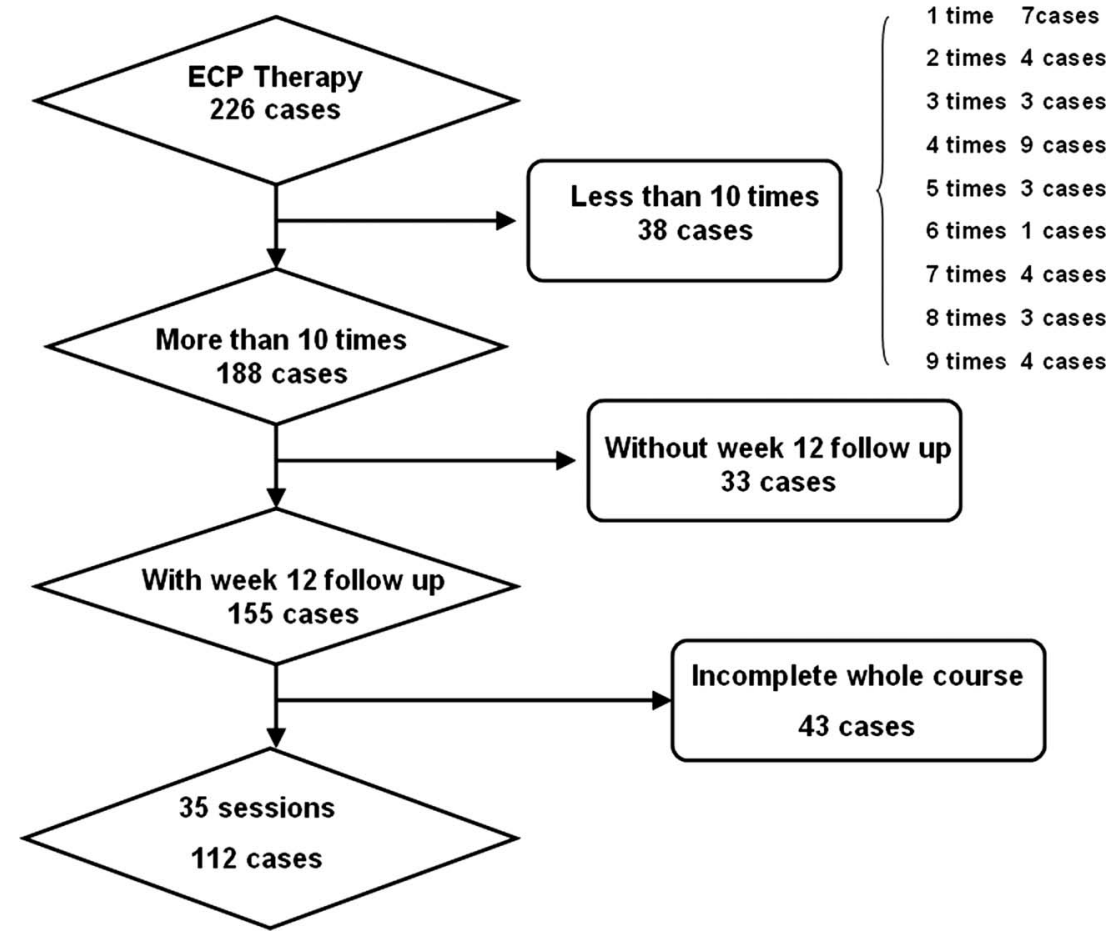

One hundred and twelve patients in the finished group completed 35 sessions, and the unfinished group had 43 patients (table 1). For $74.9 \%$ of these 207 patients, the relevant ischaemic infarct sites were located in anterior circulation, and for $22.2 \%$ of them the sites were located in posterior circulation, and $2.9 \%$ had infarcts involving both anterior and posterior circulation. There were 168 $(81.2 \%)$ patients verified with intracranial large artery stenosis, 12 patients with extracranial carotid stenosis and 27 patients with both extracranial and intracranial stenosis. For the 155 ECP-treated patients, 94 (60.6\%) received ECP treatment within 7 days after stroke onset and $144(92.9 \%)$ within 1 month. At 3 months after stroke, $70.5 \%$ of patients in the finished group had $\mathrm{mRS}$ $0-2$, compared with $46.5 \%$ in the unfinished group and $38.5 \%$ in the medical group.

Good outcome was found in $119(57.5 \%)$ patients and bad outcome in 88 patients at the 3-month follow-up. Patients in the good outcome group were younger, and had a lower baseline National Institutes of Health Stroke Scale (NIHSS), lower low-density lipoprotein cholesterol
(LDL-C) level and longer duration of ECP therapy. Patients with good outcome also had more TIA history and a slightly longer interval from stroke onset to recruitment. There was no significant difference on the use of medication between the two outcome groups (table 2).

After analysis of Multivariate logistic regression, ECP duration $(p=0.008)$, age $(p=0.023)$ and NIHSS at recruitment $(\mathrm{p}<0.001)$ remain independent predictors for a favourable functional outcome after ECP treatment (table 3). In the multivariate model, the adjusted OR associated with $1 \mathrm{~h}$ increase in ECP duration is 1.032.

For 155 patients who received ECP treatment, the distribution of median ECP duration according to the 3-month mRS scores was shown in figure 2. In 112 patients of the finished group, 55 of 63 patients (87.3\%) with a baseline mild stroke severity (baseline NIHSS $\leq 5$ ) had favourable outcome at 3 months while only 24 of 49 patients $(49 \%)$ with moderate or severe neurological impairment at baseline achieved better outcome $(\mathrm{p}<0.001)$.

Table 1 Patient group according to ECP treatment

\begin{tabular}{llll}
\hline & Medical group (52) & Unfinished ECP group (43) & Finished ECP group (112) \\
\hline Age (year) & $70.10 \pm 10.52$ & $71.47 \pm 10.51$ & $67.69 \pm 9.74$ \\
Gender, male (n, \%) & $25,48.1$ & $24,55.8$ & $78,69.6$ \\
Stroke onset to recruitment (day) & $4.46 \pm 1.79$ & $7(1-90)$ & $6(0-95)$ \\
NIHSS at recruitment & $6.5(4-16)$ & $8(0-20)$ & $5(0-22)$ \\
ECP duration (h) & 0 & $20.74 \pm 6.81$ & 35 \\
mRS 0-2 at 3 months (\%) & 38.5 & 46.5 & 70.5 \\
\hline ECP, external counterpulsation; NIHSS, National Institutes of Health Stroke Scale.
\end{tabular}


Table 2 Clinical characteristics of functional outcome groups

\begin{tabular}{|c|c|c|c|}
\hline & mRS 0-2 (119) & mRS 3-6 (88) & p Value \\
\hline Age (year) & $67.16 \pm 9.86$ & $71.67 \pm 10.07$ & 0.01 \\
\hline Gender, male (n, \%) & $78,65.5$ & $49,55.7$ & 0.085 \\
\hline $\mathrm{HT}(\mathrm{n}, \%)$ & $93,78.2$ & $68,77.3$ & 0.881 \\
\hline $\mathrm{DM}(\mathrm{n}, \%)$ & $49,41.2$ & $47,53.4$ & 0.081 \\
\hline Chronic heart disease (n, \%) & $18,15.1$ & $7,8.0$ & 0.118 \\
\hline LDL-C (mmol/L) & $2.94 \pm 1.11$ & $3.34 \pm 1.10$ & 0.014 \\
\hline HDL-C (mmol/L) & $1.25(0.70-2.40)$ & $1.24 \pm 0.33$ & 0.294 \\
\hline Triglycerides (mmol/L) & $1.50(0.40-5.40)$ & $1.40(0.60-11.70)$ & 0.306 \\
\hline Total cholesterol (mmol/L) & $4.94 \pm 1.28$ & $5.24 \pm 1.16$ & 0.09 \\
\hline Previous TIA (n, \%) & 19,16 & $3,3.4$ & 0.004 \\
\hline Previous ischaemic stroke $(n, \%)$ & 25,21 & $28,31.8$ & 0.078 \\
\hline Smoking (n, \%) & $36,30.3$ & $23,26.1$ & 0.517 \\
\hline Alcoholism (n, \%) & $15,12.6$ & $9,10.2$ & 0.597 \\
\hline Stroke onset to recruitment (day) & $6(0-95)$ & $5(0-21)$ & 0.010 \\
\hline NIHSS at recruitment & $4(0-22)$ & $9(3-20)$ & $<0.001$ \\
\hline Admission systolic BP (mm Hg) & $164.90 \pm 31.62$ & $159.16 \pm 19.22$ & 0.122 \\
\hline Admission diastolic BP $(\mathrm{mm} \mathrm{Hg})$ & $86.00(52-134)$ & $82.95 \pm 12.40$ & 0.345 \\
\hline ECP duration (h) & $35(11-35)$ & $23(10-35)$ & $<0.001$ \\
\hline Aspirin (n, \%) & $106,89.1$ & $84,95.5$ & 0.098 \\
\hline Clopidogrel (n, \%) & $11,9.2$ & $5,5.7$ & 0.343 \\
\hline ACEI $(n, \%)$ & $56,47.1$ & 51,58 & 0.121 \\
\hline$\beta$ Blocker $(n, \%)$ & $13,10.9$ & $16,18.2$ & 0.137 \\
\hline Calcium channel blocker (n, \%) & $28,23.5$ & $23,26.1$ & 0.330 \\
\hline Statin $(n, \%)$ & $90,75.6$ & $73,83.0$ & 0.203 \\
\hline
\end{tabular}

The numbers in italics are those $p$ value $<0.05$ in order to address the statistic significance.

BP, blood pressure; DM, diabetes mellitus; ECP, external counterpulsation; HDL-C, high-density lipoprotein cholesterol; HT, hypertension; LDL-C, low-density lipoprotein cholesterol; mRS, modified-Rankin scores; NIHSS, National Institutes of Health Stroke Scale; TIA, transient ischaemic attack. Continuous data were presented as the mean and SD if normally distributed or as the median and range if skew distributed.

\section{DISCUSSION}

For the first time, the duration of ECP therapy is found to be an important predictor of stroke recovery after ECP treatment. ECP duration is independently significantly associated with clinical outcome after adjustment for wellknown predictors such as age and baseline NIHSS. Patients with longer treatment duration tend to have better functional outcome 3 months after stroke. This finding is consistent with experiments in the angina pectoris, which suggests that a longer ECP duration is better. Lawson $e t a l^{12}$ reported that $21.7 \%$ of angina patients in

Table 3 Predictive factors for 3-month outcome in multivariate logistic regression

\begin{tabular}{llllr}
\hline & & \multicolumn{2}{c}{$95 \% \mathbf{C l}$} & p \\
\cline { 3 - 4 } & OR & Lower & Upper & Value \\
\hline Age (year) & 0.961 & 0.928 & 0.995 & 0.023 \\
NIHSS at recruitment & 0.734 & 0.658 & 0.819 & $<0.001$ \\
ECP duration (h) & 1.032 & 1.008 & 1.056 & 0.008 \\
LDL-C & & & & 0.155 \\
Previous TIA & & & & 0.143 \\
Stroke onset to ECP & & & & 0.075 \\
time (day) & & & & \\
\hline
\end{tabular}

The numbers in italics are those $p$ value $<0.05$ in order to address the statistic significance.

ECP, external counterpulsation; LDL-C, low-density lipoprotein cholesterol; TIA, transient ischaemic attack. an incomplete treatment course group had at least one Canadian Cardiovascular Society class reduction compared with $83.4 \%$ of patients in a complete treatment group. Data from the International EECP Patient Registry demonstrated that additional extended therapy (more than $35 \mathrm{~h}$ ) or even repeated treatment was proved to help patients achieve further symptom improvement. ${ }^{6} 1212$ The importance of ECP duration may depend on the

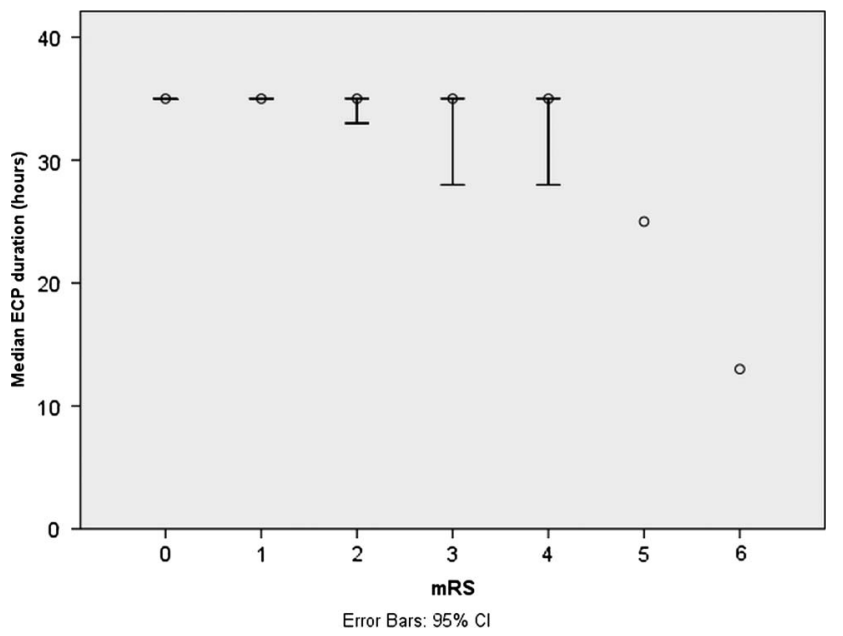

Figure 2 Distribution of median external counterpulsation duration according to the 3-month modified Rankin scores (mRS). The median and its error bar are shown. Only one patient had $\mathrm{mRS}=5$ and one patient had $\mathrm{mRS}=6$. 
mechanisms of ECP. The increased arterial wall shear stress induced by ECP in a pulsatile manner improves vascular endothelial function, which is essential to the effectiveness of ECP treatment. ${ }^{14}$ The improvement in the systemic endothelial function may promote collateral recruitment and angiogenesis, which may be affected by longer ECP through the activation and expression of vascular endothelial growth factor after $30 \mathrm{~h}$ of EECP sessions, as reported. ${ }^{15} 16$ The modified shear stress also inhibits intimal hyperplasia and atherosclerosis progression after 7 weeks of ECP, which may be due to effects of ECP on proinflammatory gene expression. ${ }^{17} 18$ Angiogenesis promotion and atherosclerosis regression caused by ECP do not occur rapidly or immediately; a long duration of treatment may be required to make a difference in these factors.

Age and baseline NIHSS are also independent predictors of clinical outcome after treatment. Age is a wellknown and important factor on stroke outcome, where lower age is associated with better outcome. ${ }^{19-21} \mathrm{~A}$ higher NIHSS score indicates a more severe stroke and predicts a poor prognosis. ${ }^{22}$ Patients with baseline mild stroke severity are more likely to benefit from a prolonged course of ECP treatment compared with those more severely impaired at baseline. Although other variables (TIA history, LDL-C level and the interval from stroke onset to recruitment) were shown to be significant in the univariate analysis, none of them was found to be independently associated with the clinical outcome in the multivariate model. Therefore, they are considered as confounders due to the relatively small sample size.

Our study has several limitations that need to be discussed. First, it is a retrospective study of a registry. Second, the number of patients included is relatively small, although this is the first report from an ECP registry for stroke patients. Third, the ECP treatment in this study is not randomised. However, there is an ongoing randomised clinical trial with the conventional medical treatment group as a control to investigate the effects of ECP on ischaemic stroke patients in our centre. The results from this randomised controlled study will be more convincing. Patients with poor outcome may find it difficult to attend ECP after discharge from the hospital, so they may be less likely to complete all 35 sessions, although our finding of the importance of treatment duration was independent of baseline NIHSS.

\section{CONCLUSION}

ECP is a new treatment concept of ischaemic stroke and we try to explore predictors of good functional outcome for ECP-treated stroke patients. We analysed our ECP registry of ischaemic stroke patients with cerebral large artery stenosis and found that duration of ECP therapy was an important predictor for good outcome after ECP treatment in addition to the well-known prognostic factors such as age and baseline NIHSS. Longer ECP duration may be associated with better clinical outcome for ECP-treated ischaemic stroke patients.
Acknowledgements This study was supported by the S. H. Ho

Cardiovascular and Stroke Center, Institute of Vascular Medicine, the Chinese University of Hong Kong.

Contributors WL collected and analysed the data and also drafted the article. $\mathrm{JH}, \mathrm{XC}$ and LX collected the data and revised the article. HWL, TWL and YS designed the study, recruited cases and revised the article. LKSW analysed the data and gave revision opinions. All authors approved this version to be published.

Funding This research received no specific grant from any funding agency in the public, commercial or not-for-profit sectors.

Competing interests None.

Patient consent Obtained.

Ethics approval Joint CUHK-NTEC Clinical Research Ethics Committee.

Provenance and peer review Not commissioned; externally peer reviewed.

Data sharing statement No additional data are available.

\section{REFERENCES}

1. Lin W, Xiong L, Han J, et al. External counterpulsation augments blood pressure and cerebral flow velocities in ischemic stroke patients with cerebral intracranial large artery occlusive disease. Stroke 43:3007-11.

2. Zheng ZS, Li TM, Kambic H, et al. Sequential external counterpulsation (secp) in china. Trans Am Soc Artif Intern Organs 1983;29:599-603.

3. Werner D, Schneider M, Weise M, et al. Pneumatic externa counterpulsation: a new noninvasive method to improve organ perfusion. Am J Cardiol 1999;84:950.

4. Manchanda A, Soran O. Enhanced external counterpulsation and future directions: step beyond medical management for patients with angina and heart failure. J Am Coll Cardiol 2007;50:1523-31.

5. Loh PH, Cleland JG, Louis AA, et al. Enhanced external counterpulsation in the treatment of chronic refractory angina: a long-term follow-up outcome from the international enhanced external counterpulsation patient registry. Clin Cardiol 2008;31:159.

6. Michaels AD, McCullough PA, Soran OZ, et al. Primer: practical approach to the selection of patients for and application of EECP. Nat Clin Pract Cardiovasc Med 2006;3:623-32.

7. Prasad GN, Ramasamy S, Thomas JM, et al. Enhanced externa counterpulsation (EECP) therapy: current evidence for clinical practice and who will benefit? Indian Heart J 2010;62:296-302.

8. Han JH, Leung TW, Lam WW, et al. Preliminary findings of external counterpulsation for ischemic stroke patient with large artery occlusive disease. Stroke 2008;39:1340.

9. Barsness G, Feldman AM, Holmes DR Jr, et al. The international EECP patient registry (IEPR): design, methods, baseline characteristics, and acute results. Clin Cardiol 2001;24:435-42.

10. Michaels AD, Linnemeier G, Soran O, et al. Two-year outcomes after enhanced external counterpulsation for stable angina pectoris (from the international EECP patient registry [IEPR]). Am J Cardiol 2004;93:461-4.

11. Felberg RA, Christou I, Demchuk AM, et al. Screening for intracranial stenosis with transcranial doppler: the accuracy of mean flow velocity thresholds. J Neuroimaging 2002;12:9-14.

12. Lawson WE, Barsness G, Michaels AD, et al. Effectiveness of repeat enhanced external counterpulsation for refractory angina in patients failing to complete an initial course of therapy. Cardiology 2007;108:170-5.

13. Michaels AD, Barsness GW, Soran $O$, et al. Frequency and efficacy of repeat enhanced external counterpulsation for stable angina pectoris (from the international EECP patient registry). Am J Cardiol 2005;95:394-7.

14. Vita JA, Mitchell GF. Effects of shear stress and flow pulsatility on endothelial function: insights gleaned from external counterpulsation therapy. J Am Coll Cardiol 2003;42:2096-8.

15. Wu G, Du Z, Hu C, et al. Angiogenic effects of long-term enhanced external counterpulsation in a dog model of myocardial infarction. Am J Physiol Heart Circ Physiol 2006;290:H248-54.

16. Gloekler S, Meier $\mathrm{P}$, de Marchi SF, et al. Coronary collateral growth by external counterpulsation: a randomised controlled trial. Heart 2010;96:202-7.

17. Zhang $\mathrm{Y}, \mathrm{He} \mathrm{X}$, Chen $\mathrm{X}$, et al. Enhanced external counterpulsation inhibits intimal hyperplasia by modifying shear stress responsive 
gene expression in hypercholesterolemic pigs. Circulation 2007;116:526-34.

18. Zhang $\mathrm{Y}, \mathrm{He} \mathrm{X}$, Liu $\mathrm{D}$, et al. Enhanced external counterpulsation attenuates atherosclerosis progression through modulation of proinflammatory signal pathway. Arterioscler Thromb Vasc Biol 2010;30:773-80.

19. Nakayama $\mathrm{H}$, Jorgensen $\mathrm{HS}$, Raaschou $\mathrm{HO}$, et al. The influence of age on stroke outcome. The copenhagen stroke study. Stroke 1994;25:808-13.
20. Hankey GJ. Long-term outcome after ischaemic stroke/transient ischaemic attack. Cerebrovasc Dis (Basel, Switzerland) 2003;16 (Suppl 1):14.

21. Wong KS, Li H. Long-term mortality and recurrent stroke risk among chinese stroke patients with predominant intracranial atherosclerosis. Stroke 2003;34:2361-6.

22. Adams HP Jr, Davis PH, Leira EC, et al. Baseline NIH stroke scale score strongly predicts outcome after stroke: a report of the trial of org 10172 in acute stroke treatment (toast). Neurology 1999;53:126-31. 